\title{
Essential attributes of a comprehensive theory of laughter
}

\author{
John Charles Simon \\ Independent scholar, United States \\ jsimon.wwl@gmail.com
}

\begin{abstract}
In principle, humour studies would be significantly enhanced by a comprehensive understanding of laughter and amusement. Members of various scientific disciplines, however, often approach laughter from distinct, rather narrow, experimental or observational paradigms, and rarely make direct challenges to broader theories that are generally accepted as incomplete, but also difficult to falsify. Evaluating, comparing, and ranking such theories is possible, however, using the concept of explanatory scope. To better establish the range of questions a comprehensive theory of laughter must resolve, I reviewed 525 peer-reviewed journal articles and recorded a total of 852 topics addressed by the authors. These were categorized into 29 themes. The list provided should allow scholars to more effectively assess the strengths and weaknesses of existing laughter theories and help guide future endeavours to more fully understand this important behaviour.
\end{abstract}

Keywords: humour, amusement, laughter theory.

\section{Introduction}

It seems improbable that inquiries into the functions and consequences of humour, however one might choose to define it, would not significantly benefit from a more comprehensive understanding of amusement. Unfortunately, of the myriad human emotions examined by philosophers and scientists over the centuries, amusement, with laughter being its most definitive outward manifestation, arguably ranks among the most poorly understood. While laughter's importance, causes, purpose, and origin have intrigued practitioners of the Western philosophical tradition as far back as ancient Greece (Halliwell 2008; Perks 2012), and continue to challenge inquisitive minds today (e.g., Bryant \& Aktipis 2014; Gervais \& Wilson 2005; Mehu \& Dunbar 2008; Owren \& Bachorowski 2003, Simon 2020), nearly all modern scholars agree that a thorough understanding of its fundamental nature continues to elude us (Apte 1985; Halliwell 2008; Kozintsev 2010; Provine 2000).

Those unfamiliar with the problem might find this conceptual shortfall surprising. After all, investigators certainly do not lack raw material, with examples of laughter available for natural history studies seemingly inexhaustible in this age of comedy clubs, fraternity 
parties, amusement parks, and now YouTube. Unlike feelings such as stress, pain, and depression, one expects few ethical constraints for clinical research procedures that generate mild anxiety or confusion, and fewer still for feelings of joy and mirth. Nor do present-day researchers want for tools. Audio and video recording equipment is readily affordable and can be employed with relative ease to allow repeated, detailed analysis of the social interactions preceding bouts of laughter, as well as the expression's outward manifestations, both in and outside laboratory settings. Many facilities possess windows into internal emotional and cognitive activities via electroencephalography, positron emission tomography, near-infrared spectroscopy, functional magnetic resonance imaging, galvanic skin response, electromyography, as well as computer-aided eye tracking, facial expression, and acoustic analyses. Perhaps of equal significance, the Internet, peer-to-peer video, and crowdsourcing software can now globalize studies, permitting larger sample populations that exhibit greater intra- and intercultural diversity than at any time in history.

Why, then, is there no single, universally accepted theory to explain laughter? From an outsider's perspective, several factors appear to be hindering efforts to either generate or authenticate an account that might reasonably be considered what Robert Provine (2000: 9) dubbed "a grand unified theory of laughter." First, inquiries into laughter have frequently been subservient to, or a by-product of, investigations into humour. This is evidenced by several international, peer-reviewed journals being devoted to the comprehension of humour whereas, regrettably, not one is dedicated to laughter. Second, explanations for laughter involve subject matter about which our understanding is constantly evolving (perception, emotion, cognition, consciousness, and communication, to name a few). And third, the mystery of laughter, like other nonverbal expressions, is being explored by a wide variety of scientific disciplines (Maryanski et al. 2013). Specialists in psychology, psychiatry, general medicine, cultural anthropology, communication, evolutionary biology, and neurology each typically use different methods to pursue different questions and then scatter published results in a wide range of journals. These investigations, often focusing on laughter's proximate causes, message(s), effects (both social and physiological), evolutionary history, and various attributes such as contagiousness or aversion (e.g., Apte 1985; Arroyo et al. 1993; Davila-Ross et al. 2009; Dezecachea \& Dunbar 2012; Hertzler 1970; Provine 1992; Ruch, Hofmann, \& Platt 2013), tend to be either largely descriptive or test hypotheses with the aim of amending or refining existing conceptual models rather than falsifying them.

Added to these hurdles is the perception by some that a comprehensive theory of laughter (or even humour) is, for all practical purposes, simply unattainable (Davies 2012; Halliwell 2008; McGee 1979). While such pessimism cannot be dismissed as wholly unjustified given the millennia-long search and the scores of explanations proposed and subsequently found wanting, it would be naïve to think such attitudes do not foster an environment that stifles innovation. As Alexander Kosintzev (2010: vii) acknowledged, "In the twenty-first century, no scholar in his/her right mind would seriously assert that s/he has come up with a brand-new and overarching theory of humour and laughter."

If we cannot eliminate from consideration existing, wide-ranging, non-falsifiable theories by making predictions and testing them directly, how can we remove weaker accounts from the pool of contenders and foster progress in this field? Are we doomed to maintain the status quo? I don't believe we are. The answer, I would suggest, involves explanatory scope. In short, relative to its competitors, how many of the agreed-upon facts established by observational and experimental studies does each theory explain?

Supporters of the five most prominent accounts (superiority, incongruity, relief, play, and attachment) each claim theirs to be dominant based on its particular strengths and its opponents' weaknesses. However, they do so absent a clear, generally-agreed-upon means of 
determining which model is the most correct. A stalemate is inevitable where no instrument of selection exists.

Here I offer a possible evaluation protocol. By compiling the various questions asked about laughter over the centuries, one can establish criteria by which various explanations might be appraised. To this end, this article seeks to answer a fundamental question: What problems must be answered and which patterns resolved for an explanation to be considered comprehensive? The conceptual model that best answers the most questions in the most parsimonious manner, as judged by knowledgeable and impartial scholars, would reign supreme until supplanted by a more comprehensive account. Over time, we would ideally converge upon a unified (and falsifiable) theory of laughter capable of answering all the questions posed. Falsifiable in that, while so-called "grand unified" theories may be effectively untestable, the answers given to specific queries typically are.

\section{Selection criteria and topic categorization}

To determine the most salient questions currently being asked by the scientific community about laughter, I performed a literature search for articles published in or between the years 1980 and 2015 containing the words laugh, laughed, laughing, or laughter in the title or subtitle. These were located with the aid of various Internet and library search engines, including a variety of journal publisher online catalogues, PsychINFO, and Google Scholar. I limited my sample to peer-reviewed journal articles and medical case reports either composed or summarized in English. To minimize the possibility of double-counting topics, I excluded articles in edited volumes, editorials, letters to editors and their replies, tributes, conference presentations or proceedings, addendums, and comments, since several might refer back to the same source article or pre-chosen topic. I omitted articles focusing on laughter yoga, laughter primarily in professional entertainment or literary contexts, and the use of laughing gas $\left(\mathrm{NO}_{2}\right)$ as a sedative, as these are extremely recent arrivals on the evolutionary scene. Also dismissed were writings about animals with the word laughing as part of their name (e.g., Laughing Thrush) and other pieces that were clearly not about laughter (e.g., titles suggesting an unrelated subject was "no laughing matter").

I attempted to review as many articles fitting these criteria as was practical. As new titles became markedly difficult to find, I settled upon the otherwise arbitrary goal of 525. For most ( $n=470)$ I was able to obtain the full text. For the remainder I had access to only the abstract (including articles composed in languages other than English). Because I was interested in the principal topics raised within each article, I predominantly gleaned those mentioned in the abstract. When abstracts were not provided $(n=28$; typically medical case reports), I surveyed the introduction, the entire report, or the entire article as needed. I logged up to three topics per article.

I assessed the various topics or questions raised within each article and grouped them together into general themes (e.g., laughter and emotional coping, laughter's influence on social relationships, and laughter's evolutionary origins). For example, if five articles each raised the topic of contagiousness and two articles each raised the topics of smiling and play, a total of nine topics were tallied, but they would be grouped into three general themes: contagiousness, smiling, and play. I read through each abstract or article twice, initially assigning each topic to a particular theme and then, in the second round, reassigned them if they better fit a classification first encountered after the initial appraisal. 


\section{Findings}

I recorded a total of 852 topics addressed in the 525 reviewed articles, with 252,219 , and 54 articles raising one, two, and three topics, respectively. I distributed each to one of 29 different themes. A summary of the findings are presented in Table 1.

Table 1. A list of 29 laughter-related themes and the number of times they were addressed in all 525 reviewed articles. Total count is 852 .

\begin{tabular}{|c|c|c|}
\hline Count & Per cent of Total & Theme \\
\hline 121 & 14.0 & Effects of Emotional or Cognitive Disability/Injury \\
\hline 91 & 10.7 & Social Influences, Group Dynamics, Information Transfer \\
\hline 70 & 8.2 & Laughter and the Brain \\
\hline 65 & 7.6 & Physical Effects and Benefits \\
\hline 62 & 7.3 & Emotional Coping \\
\hline 53 & 6.2 & Variation in the Laugh Response \\
\hline 47 & 5.5 & Relationship with Humour \\
\hline 42 & 4.9 & Evolutionary Function \\
\hline 38 & 4.5 & Misinterpreting the Laugher's Intent \\
\hline 35 & 4.1 & Acoustic Structure and Vocal Qualities \\
\hline 31 & 3.6 & Message Conveyed \\
\hline 25 & 2.9 & Indicators of Personality, Health, and Status \\
\hline 24 & 2.8 & Relationship with Smiling \\
\hline 23 & 2.7 & Laughter's Dual Nature (Laughing With vs $A t$ ) \\
\hline 21 & 2.5 & Evolutionary Origins \\
\hline 13 & 1.5 & Using Laughter Volitionally \\
\hline 12 & 1.4 & Laughter's Contagious Quality \\
\hline 12 & 1.4 & Varieties of Laughter \\
\hline 8 & 0.9 & Variations in Intensity/Duration \\
\hline
\end{tabular}




\begin{tabular}{|c|c|c|}
\hline 8 & 0.9 & Laughter and Humor's Use by Bullies \\
\hline 7 & 0.8 & Relationship with Tickling \\
\hline 7 & 0.8 & What we mean by a "Sense of Humuor" \\
\hline 6 & 0.7 & Laughter as an Instinctive vs Learned Behaviour \\
\hline 6 & 0.7 & Computational Laughter and Humour \\
\hline 5 & 0.6 & Relationship to Crying (Sobbing) \\
\hline 4 & 0.5 & Laughing at Ourselves \\
\hline 4 & 0.5 & Laughter as a Response to Positive Realizations \\
\hline 4 & 0.5 & Evolution of Laughter or the Laugh Response \\
\hline 3 & 0.4 & Laughter's Relationship to Play \\
\hline 2 & 0.2 & Influence of Drugs on the Laugh Response \\
\hline 2 & 0.2 & Laughing in Response to Animals/Inanimate Objects \\
\hline 1 & 0.1 & Inability to Tickle Oneself into Laughter \\
\hline
\end{tabular}

\section{Discussion}

Several self-imposed constraints prevented this review from being exhaustive. As a monoglot, I was limited to articles providing at least an abstract in English. Also excluded were treatments of this subject that did not contain some derivation of the word laugh in their titles (i.e., humour, amusement, or mirth). Because of uncertainty in the acceptance criteria, I refrained from sampling papers contributed to edited volumes. Furthermore, there may have been compelling questions asked by scholars prior to 1980 that are no longer of great interest to investigators publishing in or after that year. Still, I contend that the articles sampled, drawn from a staggering 345 different scientific and medical journals, relate the vast majority of topics raised in this domain.

Categorization, by definition, produces a simplified portrayal of the original population, and this study is no exception. As in other disciplines, there are experts who are "lumpers" and those who are "splitters." I chose to separate themes as much as was practical and allow the reader to combine similar concepts as he or she sees fit. For example, the observation that even a highly ticklish individual cannot, as a general rule, tickle him- or herself into a bout of genuine laughter (raised by only one author in my sample) could easily have been lumped with other topics examining tickle-inspired laughter. However, it seemed a sufficiently intriguing and widespread phenomenon to necessitate its own dedicated explanation. On the other end of the spectrum, variation in the laugh response is such a diverse 
and complex theme that splitting it into subheadings (e.g., differences related to age, life experience, culture, sex, religious or political views, social context, mood, etc.) would have required a rather extensive article unto itself. For our purposes, it is enough to recognize that any comprehensive theory of laughter would need to account for all such variability.

Two of the most commonly addressed themes, justifiably of interest to many, can nevertheless be considered peripheral to the understanding of laughter as a behaviour. Studies of responses in individuals with cognitive and/or emotional disabilities such as gelastic epilepsy, a common focus of numerous medical case reports (e.g., Holmes \& Goldman 2012), or gelatophobia (e.g., Ruch et al. 2015) offer little insight into function and consequences of laughter in our species as a whole. Evaluating the abnormal assumes one has some fundamental understanding of the normal. Similarly, knowing the exact neural pathways that generate the vocalization we recognize as laughter (e.g., Citardi et al. 1996; Jefferson 2010; Szameitat et al. 2011) may reveal how we produce it, but says nothing about why. Less popular topics also have minimal impact on our understanding of this behaviour. Computational humour, the recognition and production of humour and laughter using artificial intelligence, is a field that would no doubt benefit from an increased knowledge of laughter's proximate causes, but arguably contributes only marginally to that endeavour.

Combining some of the more closely related themes presented in Table 1, the following are 23 problems that should be coherently explained by a comprehensive theory of laughter:

1. Function. Did laughter principally evolve as a reflexive response to certain stimuli, a physiological adaptation for relieving tension or stress, a nonconscious expression of an internal emotional state, an active (conscious) form of communication, or something else entirely?

2. Instinctive. To what extent is laughter an instinctive versus learned behaviour?

3. Message. If laughter is communication, what message(s) does it convey and why has it not been displaced by verbal language?

4. Origins and Evolution. With regard to its evolutionary origins, do other animals, the great apes in particular, display "true" laughter that is functionally equivalent to ours or, instead, some type of proto-laughter? If the former, why has laughter's use by humans increased in frequency and scope from what we observe in the great apes?

5. Varieties. Are there different varieties of laughter: nervous, embarrassed, mocking, sympathetic, exultant, revealing, etc.? If there are, does each have a different message or convey a different emotional state?

6. Duality. How do we account for laughter's dual nature, in that most people recognize a qualitative difference between laughing sympathetically with someone and laughing derisively at someone, even if they cannot always articulate this difference? Which is the default, or most common, motivation?

7. Intensity and Duration. Why do bouts of laughter vary in intensity and duration?

8. Variation. How can we explain observed differences in the laugh response - within the same individual over short and long timescales; as a function of relationship, social context, mood, and health (including the effects of drugs); as well as between individuals, cultures, age groups, sexes, etc.?

9. Humour. What is humour and its relationship with laughter? To what do we typically refer when we suggest someone has a good sense of humour or a poor one?

10. Sociality. How and why do laughter and humour influence group composition, group dynamics, and the uptake and/or transfer of knowledge?

11. Bullying. Why are laughter and humour often used as weapons by bullies? 
12. Coping and Health. How and why does laughter (or amusement) bolster our emotional resilience and well-being?

13. Misinterpretation. Why, if we typically delight in the laughter of our friends and peers, do we sometimes (for gelotophobes, often) find it distressing, apparently misinterpreting the laugher's intent?

14. Smiling. Why are expressions of laughter so closely associated with smiling?

15. Crying. What is laughter's relationship to crying (sobbing), especially in its physical manifestation, and why do we occasionally laugh and cry at the same time?

16. Structure. Why does laughter have the distinctive vocal properties that it does?

17. Volitional. Why do we sometimes purposefully feign, exaggerate, or inhibit our laughter?

18. Contagiousness. Why is laughter sometimes, but not always, contagious?

19. Tickling. Why do many people laugh when tickled by another individual and some rarely or never do? Why can we not, generally speaking, tickle ourselves into a bout of sincere laughter?

20. Play. What explains laughter's strong association with play, both in humans and great apes?

21. Self-Reflection. Why do we sometimes laugh in response to our own misfortune or shortcomings in addition to those of others?

22. Positive. Why do we sometimes laugh in response to our own and others' good fortune and triumphs, e.g., clever remarks or displays of exceptional skill?

23. Non-anthropogenic. Why do we sometimes laugh in response to nonhuman animals and even inanimate objects?

In addition to those specifically raised by the reviewed authors, I would argue these three questions should also be tackled:

24. Solo. Why do we sometimes laugh even when we are alone (Hertzler 1970; Provine 2000)?

25. Delight. Why do we typically experience a positive affect when we engage in the act of laughing (Dunn 1994; Nelson 2012; Provine 1996)?

26. Amusement. How can we best understand the sensation we refer to as amusement (or mirth) and explain its relationship with the behaviour of laughter (Morreall 2015; Roberts 1988; Simon 2008)?

\subsection{Comprehensiveness}

The themes listed above include the four fundamental questions scholars ask about a behaviour (Chiszar 1981; Martin 1984). In this context, first, a complete theory must provide an understanding of laughter's function. For what purpose is it generated? Second, it needs to explain laughter's development over the course of an individual's lifetime, from infancy through adulthood (see Variation). Third, it should reveal the process by which laughter is elicited, its proximate causation. And finally, it must provide a logical premise for laughter's ultimate causation, i.e., its evolutionary utility and design. Why is it that we should engage in this behaviour at all, and why does it take the form of this particular vocalization instead of, say, a whistle, a wrinkling of the nose, or the snapping of fingers?

Beyond these minimum requirements, we should expect a truly comprehensive model to answer all of the remaining questions that have been asked about laughter. Why is it closely associated with smiling? Why is it contagious in some circumstances and not in others? Why 
are some people ticklish, but not others - and why can't we tickle ourselves into fits of sincere laughter? The ability to coherently address a wide range of seemingly unrelated, yet wellestablished facts is a function of the explanatory scope of a theory (Johnson et al. 2014).

\subsection{Parsimony}

Theorists seek explanations that are parsimonious as well as complete. Assuming both are consistent with evolutionary principals, if two conceptual models answer the relevant questions equally well, the simpler theory is generally given precedence. What does this mean with regard to laughter? Some examples come to mind.

For those advocating a fundamental link between the laughter of the great apes and humans, the theory must perform as well with that brought about by tickling, chasing, and rough-and-tumble play as it does by jokes, riddles, sarcasm, and irony. Moreover, to conclude that there are many different "types" of laughter (e.g., Mahony et al. 2002; Ruch et al. 2013) is likewise less parsimonious than to purport only a single function for this behaviour.

When laughter is advanced primarily as communication, ascribing to it a single message is clearly more economical than multiple ones and consistent with Darwin's view that natural selection would not favour the development of such confusing signals (Owings \& Morton 1998). For example, suggesting, as some do, that laughter signals an expression of current superiority over another as well as a sender's desire to, at some future time, establish or return to such a state of superiority represents two quite different, some might say mutually exclusive, notions. A singular message, albeit one that might be construed in either a positive or negative light given various receivers and contexts, will be the more efficient paradigm.

\subsection{Testability}

For a model to qualify as a viable scientific theory, it must be testable, or more accurately, falsifiable. It must lend itself to experiments specifically designed to refute the proposed theoretical construct. Testability implies measurability. Existing concepts such as superiority, incongruity, and tension relief, to name only a few, can be often difficult to describe, let alone quantify. A successful theory will be one having central components that are well-defined, specific, consistent with accepted evolutionary principals, and measurable. This is not to say that key elements may not also be subjectively gauged. Given the variability of the laugh response, they almost certainly will be. However, an explanation cannot be so malleable as to make it useless as a predictive tool (Deutsch 1997).

\section{Conclusion}

The results of this study suggest that many of the questions faced in the last two millennia are still plaguing laughter scholars today. Edmond Bergler (1956: 31) suggested that theorists "cannot agree on the reasons and mechanisms that produce laughter, and therefore they produce innumerable theories which by and large magnify one aspect of the problem, mistakenly taking it for the essential." This appears as true now as it was then.

The list of questions presented ought not be considered final or complete. Others interested in this behaviour should provide alternatives and make the case for subtracting, adding, and combining elements as they deem necessary. However, to make significant headway in the quest to fully understand laughter, reason dictates we create an environment in which all theories are competing on a level, well-delineated playing field. We should establish a rule-based competition where lesser theories can be respectfully set aside and new models 
are encouraged to participate. Such selective processes are crucial for advancements within all scientific disciplines.

\section{Acknowledgements}

The author wishes to thank two anonymous reviewers for providing critical feedback for improving an earlier draft of this manuscript.

\section{References}

Apte, M. (1985). Humour and Laughter: An Anthropological Approach. Ithaca: Cornell University Press.

Arroyo, S., Lesser, R. P., Gordon, B., Uematsu, S., Hart, J., Schwerdt, P., Andreasson, K., \& Fisher, R. S. (1993). 'Mirth, laughter and gelastic seizures'. Brain 116, pp. 757-780.

Bergler, E. (1956). Laughter and the Sense of Humour. New York: Intercontinental Medical Book Co.

Bryant, G. A., \& Aktipis, C. A. (2014). 'The animal nature of spontaneous human laughter'. Evolution and Human Behaviour 35, pp. 327-335.

Citardi, M. J., Yanagisawa, E., \& Estill, J. (1996). 'Videoendoscopic analysis of laryngeal function during laughter'. Annals of Otology, Rhinology and Laryngology 105, pp. 545549.

Chiszar, D. (1981). 'Learning theory, ethological theory, and developmental plasticity', in E. S. Gollin (ed.), Developmental Plasticity: Behavioral and Biological Aspects of Variations in Development. New York: Academic Press, pp. 71-100.

Davies, C. (2012). Book Reviews. Humour: International Journal of Humour Research 25(1), pp. 108-113.

Davila-Ross, M., Owren, M., \& Zimmermann, E. (2009). 'Reconstructing the evolution of laughter in great apes and humans'. Current Biology 19(13), pp. 1106-1111.

Deutsch, D. (1997). The Fabric of Reality: The Science of Parallel Universes. New York: Allen Lane.

Dezecachea, G., \& Dunbar, R. I. M. (2012). 'Sharing the joke: The size of natural laughter groups'. Evolution and Human Behaviour 33, pp.775-779.

Dunn, J. (1994). 'Experience and understanding of emotions, relationships, and membership in a particular culture', in P. Eckman and R. J. Davidson (eds.), The Nature of Emotion: Fundamental Question. New York: Oxford University Press, pp. 352-355.

Gervais, M., \& Wilson, D. S. (2005). 'The evolution and functions of laughter and humour: A synthetic approach'. Quarterly Review of Biology 80, pp. 395-430.

Halliwell, S. (2008). Greek Laughter: A Study Of Cultural Psychology From Homer To Early Christianity. Cambridge University Press.

Hertzler, J. O. (1970). Laughter: A Socio-Scientific Analysis. New York: Expedition Press.

Holmes, C. M., \& Goldman, M. J. (2012). 'Seizures presenting as incessant laughter: A case of gelastic epilepsy'. The Journal of Emergency Medicine 43(6), pp. e447-e449.

Jefferson, G. (2010). 'Sometimes a frog in your throat is just a frog in your throat: Gutturals as (sometimes) laughter-implicative'. Journal of Pragmatics 42(6), pp. 1476-1484.

Johnson, S. G., Johnston, A., Toig, A., \& Keil, F. (2014). 'Explanatory scope informs causal strength inferences'. Cognitive Science 38, pp. 2453-2458. 
Kozintsev, A. (2010). The Mirror of Laughter. Translated by Richard P. Martin. New Brunswick and London: Transaction Publishers.

Mahony, D. L., Burroughs, W. J., \& Lippman, L. G. (2002). 'Perceived attributes of healthpromoting laughter: A cross-generational comparison'. The Journal of Psychology 136(2), pp. 171-181.

Martin, P. (1984). 'The (four) whys and wherefores of play in cats: A review of functional, evolutionary, developmental and causal issues', in P. K. Smith (ed.), Play in Animals and Humans. New York: Basil Blackwell, pp. 71-94.

Maryanski, A. M., Molnár, P., Segerstråle, U., \& Velichkovsky, B. M. (2013). 'The social and biological foundations of human communication', in P. Weingart, S. D. Mitchell, P. J. Richerson, \& S. Maasen (eds.), Human by nature: Between biology and the social sciences. Psychology Press, (pp. 181-200).

Mehu, M., \& Dunbar, R. I. M. (2008). 'Naturalistic observations of smiling and laughter in human group interactions'. Behavior 145, pp. 1747-1780.

Morreall, J. (2015). 'Is humorous amusement an emotion?' The Israeli Journal of Humor Research 4(2), pp. 6-11.

Nelson, J. K. (2012). What Made Freud Laugh: An Attachment Perspective on Laughter. New York: Routledge.

Owings, D. H., \& Morton, E. S. (1998). Animal Vocal Communication: A New Approach. Cambridge, England: Cambridge University Press. [referring to Darwin, C. (1872). The Expression Of the Emotions in Man and Animals. London: Murray.]

Perks, L.G. (2012). 'The ancient roots of humour theory'. Humour: International Journal of Humour Research 25(2), pp. 119-132.

Provine, R. R. (1992). 'Contagious laughter: Laughter is a sufficient stimulus for laughs and smiles'. Bulletin of the Psychonomic Society 30(1), pp. 1-4.

Provine, R. R. (1996). 'Laughter'. American Scientist 84, pp. 38-44.

Provine, R. R. (2000). Laughter: A scientific investigation. New York, NY: Penguin Press.

Roberts, R. C. (1988). 'Is amusement an emotion?' American Philosophical Quarterly 25(3), pp. 269-274.

Ruch, W. F., Hofmann, J., \& Platt, T. (2013). 'Investigating facial features of four types of laughter in historic illustrations'. The European Journal of Humour Research 1(1), pp. 99-118.

Ruch, W. F., Hofmann, J., \& Platt, T. (2015). 'Individual differences in gelotophobia and responses to laughter-eliciting emotions'. Personality and Individual Differences 72, pp. 117-121.

Simon, J. C. (2008). Why We Laugh: A New Understanding. Indianapolis, Indiana: Starbrook Press.

Simon, J. C. (2020). 'Laughter Redefined'. The Israeli Journal of Humor Research 9(1).

Szameitat, D. P., Darwin, C. J., Szameitat, A. J., Wildgruber, D., \& Alter, K. (2011). 'Formant characteristics of human laughter'. Journal of Voice 25(1), pp. 32-37. 